\title{
Téoros
}

Revue de recherche en tourisme

\section{La prospective : méthodes et outils}

\section{Fabrice Roubelat}

Volume 12, numéro 2, juillet 1993

La prévision-prospective du tourisme

URI : https://id.erudit.org/iderudit/1078019ar

DOI : https://doi.org/10.7202/1078019ar

Aller au sommaire du numéro

\section{Éditeur(s)}

Université du Québec à Montréal

\section{ISSN}

0712-8657 (imprimé)

1923-2705 (numérique)

Découvrir la revue

Citer cet article

Roubelat, F. (1993). La prospective : méthodes et outils. Téoros, 12(2), 7-9. https://doi.org/10.7202/1078019ar d'utilisation que vous pouvez consulter en ligne.

https://apropos.erudit.org/fr/usagers/politique-dutilisation/ 


\title{
La prospective: méthodes et outils
}

\author{
Fabrice Roubelat"
}

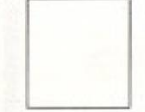

\section{Prospective et futuribles}

Àla fin des années cinquante, les pères fondateurs de l'école française de prospective donnaient naissance à ce que Pierre Massé allait qualifier d'windiscipline intellectuelles. Concept créé par Gaston Berger cependant que Bertrand de Jouvenel proposait celui de futuribles, la prospectiveest devenue synonyme de l'étude du futur.

Résolument préactive et proactive, la prospective cherche à bâtir un futur désiré tout en tenant compte des multiples contraintes de l'environnement. Elle allie toutefois cette attitude volontariste à l'idée que l'avenir est multiple et indéterminé, composé de futurs possibles, les futuribles.

Durant les années soixante-dix, cette école a peu à peu assimilé les différents outils développés outre-atlantique dans le domaine des études sur le futur: méthode Delphi, impacts croisés quantitatifs et qualitatifs sont ainsi venus enrichir une démarche encore en gestation. Sous l'impulsion de Michel Godet, ces outils ont été perfectionnés, standardisés et surtout complétés de manière à former une boîte à outils, aujourd'hui informatisée, opérationnelle et appropriable.

Intégrée, la boîte à outils de la prospective constitue également le support d'une approche maintenant devenue classique: la méthode des scénarios.

\section{Une démarche intégrée : la méthode des scénarios}

Lorsque l'on parle de prospective, chacun pense immédiatement à l'outil de base que constituent les scénarios. Or, comme le fait très justement remarquer Michel Godet, scénarios et prospective ne sont synonymes. En effet, pour être réellement prospectifs, les scénarios se doivent de respecter des critères de pertinence, de cohérence et de vraisemblance. Pour ce faire, la recherche des futurs possibles doit s'accompagner d'un minimum de méthode.
Intégrantnombre d'outils dela prospective, Michel Godeta dès les années soixante-dix élaboré une méthode complète de construction des scénarios reprenant les étapes suivantes:

- délimitation du système et recherche des variables-clés (analyse structurelle);

- rétrospective et analyse du jeu des acteurs (Mactor);

- probabilisation deshypothèses-cléset de leur combinaison (Smic-ProbExpert);

- recherche des images-clés du futur (analyse morphologique);

- choix des options stratégiques (méthodes multicritères).

Comme le montre la dernière étape, cette démarches'articuleaveclesoutils classiques de la stratégie que sont par exemple les méthodes multicritères. Elle a d'ailleurs fait l'objet d'une approche intégrant prospective et stratégie en utilisant des outils comme les arbres de compétence de Marc Giget.

En fait, le but de ces phases successives est de construire des scénariosincluantà la fois des images et des cheminements, et de prendre en considération les jeux d'acteurs, tout en respectant les critères ếnoncés plus haut.

Chacune des phases de la méthode des scénarios s'appuie donc sur des outils formalisés que l'on peut en outre mettre en oeuvre séparément et que nous allons brièvement passer en revue.

\section{À la recherche des variables-clés: I'analyse structurelle}

Mettre en évidence les variables-clés en se posant les bonnes questions et en ne négligeant aucune piste, tel est l'objectif de l'analyse structurelle. En effet, dans toute prospective, il est d'abord essentiel de distinguer, parmi les multiples variables influençant le système, tendances lourdes et 


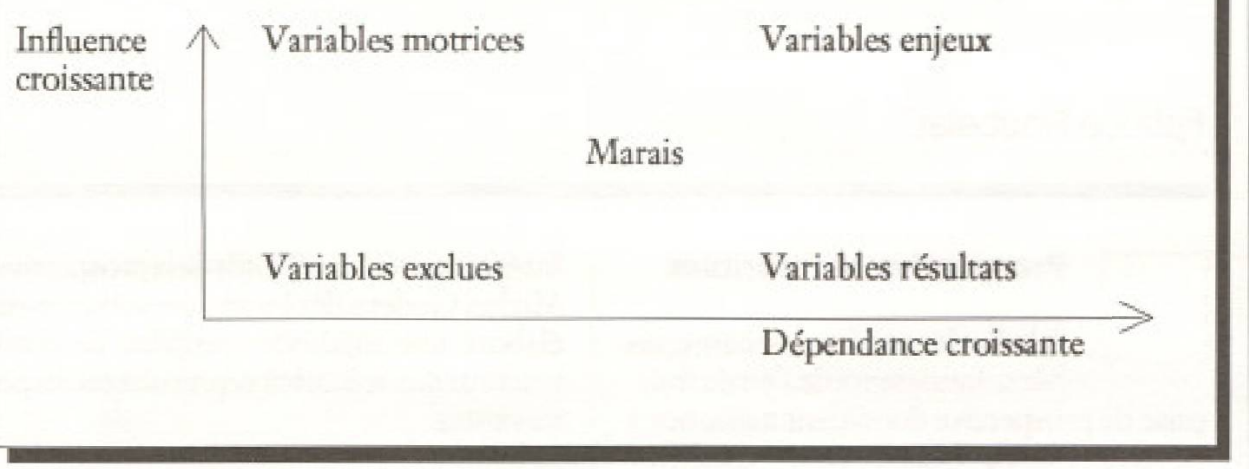

variables de rupture, enjeux, résultats et faux problèmes.

A cette fin, l'analyse structurelle est une technique d'analyse de système qui permet de décrire le domaine étudié en procédant en trois étapes successives:

- identifier les composantes du système, ainsi que les relations entre ces composantes;

- exposer le fonctionnement du système et en déterminer les variablesclés(tendances lourdes et variables de rupture);

- esquisser les évolutions du système.

La première étape donne lieu à la construction d'une matrice qualitative d'interactions (matrice d'analyse structurelle). A partir de cette matrice, on calcule des indicateurs d'influence et de dépendance à la fois directs (relations d'ordre 1) et indirects (prise en compte des relations indirectes et notamment les boucles de rétroaction). Le dassement indirect est obtenu par le programme Micmac qui consiste tout simplement à élever la matrice en puissance. Ces indicateurs permettent de construire le diagramme influence-dépendance, qui dresse une typologie des variables.

Quelles qu'elles soient, toutes ces variables assurent une fonction dans le système, y compris les variables exclues qui sont souvent des thèmes de communication permettant de masquer les vrais problèmes. La forme du nuage de points constitue quant à elle un indicateur de stabilité riche d'enseignements sur la dynamique du système, tout comme la comparaison entre les diagrammes directs et indirects.

\section{L'analyse des jeux d'acteurs : la méthode Mactor}

Dans un environnement toujours plus instable, les jeux et stratégies d'acteurs prennent une part prépondérante dans la construction de scénarios. Ainsi, autour des variables-clés dégagées lors de l'analyse structurelle, il convient de mettre un peu de chair et de distinguer les stratégies des acteurs qui s'y affrontent dans des jeux incertains et complexes.

La méthode Mactor s'efforce de visualiser, autour d'un ensemble d'objectifs, les convergences et divergences entre acteurs, tout en prenant en compte leurs rapports de forces. Alors, il devient possible de mettre en lumière les enjeux stratégiques de ceux qui le sont moins et d'apprécier les issues des alliances et conflits à venir.

Pour ce faire, Mactor utilise les données issues des deux matrices suivantes:

- MID, matrice d'influence directe entre acteurs: comment chaque acteur peut-il influencer chaque autre (existence, mission, projets, processus opératoires)?

- MAO, matrice acteurs/objectifs: niveau de l'impact de la réalisation de l'objectif sur l'acteur (existence, mission, projets, processus opératoires)?

À partir deces deuxtypes d'entrées, Mactor permet de calculer des matrices et de construire des graphes de convergences $(\mathrm{CAA})$ et de divergences $(\mathrm{DAA})$, pondérées par les rapports de forces indirects $(\mathrm{MII}=$ MID x MID) entre acteurs.

Ainsi, cette méthode dégage, en fonction des alliances et conflits potentiels qu'elle visualise, les acteurs et objectifs-clés.
La principale qualité d'une méthode comme Mactor est de reposer sur des principes simples. En ce sens, elle est facilement appropriable par les groupes de travail qui choisissent de la mettre en oeuvre. Le recours aux matrices acteurs/objectifs en fait un outil qui a d'emblée une signification claire et apporte des réponses presque immédiates aux préoccupations de ces groupes. Defait, l'analyse de jeux d'acteurs tend parfois à remplacer l'analyse structurelle en tant qu'outil utilisé de manière autonome.

\section{Probabiliser les futurs : les méthodes de consultation d'experts}

Outils de quantification, les méthodes d'experts ont principalement pour objectif de recueillir des estimations par l'intermédiaire de jugements d'experts et de les combiner afin d'élaborer des scénarios.

Ces estimations se répartissent en trois catégories:

- la probabilité de réalisation d'un événement ou d'une hypothèse: quelle est la probabilité qu'un accident nucléairegrave survienne d'icil'an 2000?

- la date de réalisation d'un événement: en quelle année l'homme marcherat-il sur Mars?

- la valeur d'un paramètre: quel sera le taux de croissance du PIB en 1995?

Les techniques de consultation d'experts appliquées à la prospective sont sans aucun doute les outils qui ont fait l'objet des tentatives de formalisation les plus variées et sophistiquées.

Développée dans les années cinquante aux Etats-Unis à la Rand Corporation par Olaf Helmer et Norman Dalkey, la méthode Delphi a pour objectif de déterminer des estimations quantifiées en faisant converger les réponses des différrents experts. Elle suppose donc une corrélation entre la valeur de l'estimation et le nombre d'experts en accord avec l'estimation en question. Ainsi, à la question de la date probable de la réalisation de croisières sur la Lune, on cherchera, par l'intermédiaire de plusieurs tours (consultations postales ou informatiques), à concentrer les réponses des experts dans l'intervalle le plus réduit possible. 
Depuis le développement de Delphi, les technologies de l'information sont venues structurer le processus de communication visant à obtenir de telles estimations: machines à voter (consensor ou abaque de Régnier) etmaintenantles groupruares(outils d'aide au travail coopératif).

Plusélaborées sont les méthodes d'impacts croisés quantitatifs qui privilégient le traitement des données obtenues. Ainsi, à partir d'un système d'hypothèses (occurrence d'événements et états des variablesclés), ces méthodes permettent de calculer des probabilités finales tenant compte de l'interdépendance des hypothèses. En outre, desoutils comme Smic-Prob-Expert s'attachent à Probabiliser les scénarios issus de la combinaison de ces hypothèses et permettent ainsi de balayer le champ des possibles afin de dégager des scénarios qui, bien que parmi les plus probables, sont souvent écartés au cours d'études moins systématiques.

\section{Maîtriser la combinatoire: I'analyse morphologique}

Élaborée dans les années quarante par le mathématicien $\mathrm{F}$. Zwicky pour faire de l'invention une routine, l'analyse morphologique, fortement liée au départ à la prévision technologique, est aujourd'hui utilisée de manière beaucoup plus large. La méthode consiste à décomposer le système étudié en sous-systèmes, puis en composantes. Ainsi, si un menu propose 3 entrées, 4 plats, 3 desserts et 5 boissons, le nombre de configurations possibles est de $3 \times 4 \times 3 \times 5$, c'est-à-dire 180 solutions.

La combinaison des composantes ainsi listées permet doncdedisposer d'un champ de configurations important. Pour ne pas être noyé par la combinatoire, il convient alors de fixer des facteurs d'exclusion et de préférence à partir desquels les solutions seront triées.

Ainsi, l'analyse morphologique se prête tout à fait à la construction de scénarios et d'images comme combinaisons d'hypothèses. Par exemple, des scénarios globaux peuvent présenter des composantes économiques, sociales, politiques, technologiques dont la combinaison des états possibles (pas d'intégration européenne, intégration à 12, intégration élargie pour une composante liée à l'intégration euro- péenne) donne de nombreuses images du futur.

\section{Conjuguer rigueur et communication}

Une des particularités principales de la prospective est, pour construire ses modèles et calculer ses estimations, de se baser sur des données qui, quantitatives comme qualitatives, restent d'abord subjectives. Aussi, les outils mathérnatiques proposés peuvent être aussi sophistiqués qu'on le voudra, ils ne feront que transformer des données qui, parfois, seront sujettes à caution. Aussi, cet aphorisme d'informaticien qu'est *garbage in, gospel out exprime parfaitement l'état d'esprit qui doit animer l'utilisation des outils que nous venons de pré́senter.

En particulier, leur mise en oeuvre ne peut en aucun cas être mécanique et doit reposer sur leur appropriation par lesutilisateurs. Cette appropriation est d'autant plus importante que la plupart de ces outils ne sont que des «coquilles à remplirs: et la coquille n'est bonne que par ce que l'on met à l'intérieur (toujours notre aphorisme). Quant au fumer qu'elle dégage, il dépend en grande partie du processus de communication que l'outil contribueà enclencher: comme le proclame Michel Godet, la prospective n'est pas un plaisir solitaire! Toutefois, on devra se garder de sacrifier la rigueur des outils à un exercice de communication qui risque alors de transformer la prospective en entertainment. $f$

\section{Bibliographie}

GODET. M., De Fanticlpation ta Faction, manuel de prospective et de strategie, Dunod, 1992. Traduction anglaise: From Anticipation to Action, UNESCO, 1993.

ROUBELAT, F, L'analyse structurelle, L'analyse des jeux d'acteurs. Les methodes de consultation d'experts, dans La prospective: histoire, pratiques et móthodes, sous la direction de Fabrice Hatem, Economica, 1993

Les deux ourrages ci-dessus fourniront au lecteur une bibliographie plus complete. 